This is a postprint version of the following published document:

Liu, Yanfang; Olmos, Pablo M.; Koch, Tobias. On LDPC code ensembles with generalized constraints, in: 2017 IEEE International Symposium on Information Theory (ISIT) [Proceedings], pp. 371-375, August 2017

DOI: https://doi.org/10.1109/ISIT.2017.8006552

C2017 IEEE. Personal use of this material is permitted. Permission from IEEE must be obtained for all other uses, in any current or future media, including reprinting/republishing this material for advertising or promotional purposes, creating new collective works, for resale or redistribution to servers or lists, or reuse of any copyrighted component of this work in other works. 


\title{
On LDPC Code Ensembles with Generalized Constraints
}

\author{
Yanfang Liu, Pablo M. Olmos, Tobias Koch \\ Universidad Carlos III de Madrid \& Gregorio Marañón Health Research Institute \\ Email: $\{$ vivian, olmos, koch\}@tsc.uc $3 m$.es
}

\begin{abstract}
In this paper, we analyze the tradeoff between coding rate and asymptotic performance of a class of generalized lowdensity parity-check (GLDPC) codes constructed by including a certain fraction of generalized constraint (GC) nodes in the graph. The rate of the GLDPC ensemble is bounded using classical results on linear block codes, namely Hamming bound and Varshamov bound. We also study the impact of the decoding method used at GC nodes. To incorporate both bounded-distance (BD) and Maximum Likelihood (ML) decoding at GC nodes into our analysis without having to resort on multi-edge type of degree distributions (DDs), we propose the probabilistic peeling decoder (P-PD) algorithm, which models the decoding step at every GC node as an instance of a Bernoulli random variable with a success probability that depends on the GC block code and its decoding algorithm. The P-PD asymptotic performance over the BEC can be efficiently predicted using standard techniques for LDPC codes such as density evolution (DE) or the differential equation method. Furthermore, for a class of GLDPC ensembles, we demonstrate that the simulated P-PD performance accurately predicts the actual performance of the GLPDC code. We illustrate our analysis for GLDPC code ensembles using $(2,6)$ and $(2,15)$ base DDs. In all cases, we show that a large fraction of GC nodes is required to reduce the original gap to capacity.
\end{abstract}

Index Terms-Generalized low-density parity-check codes, codes on graphs, peeling decoding

\section{INTRODUCTION}

Generalized low-density parity-check (GLDPC) block codes were first proposed by Tanner. In contrast to standard LDPC codes, which are represented by bipartite Tanner graphs where variable nodes and single parity-check (SPC) nodes are connected according to a given degree distribution (DD), in GLDPC codes the SPC nodes in the graph are replaced by generalized constraint (GC) nodes [1]. The sub-code associated to each GC node is referred to as the component code. Examples of component codes used in the GLDPC literature are Hamming codes [2], Hadamard codes [3] or expurgated random codes [4]. Upon selecting a particular class of component codes, the DD of the GLDPC code ensemble can be optimized, and nearcapacity iterative decoding thresholds can be achieved [2], [4], [5]. Capacity-achieving GLDPC code ensembles can also be

This work has been funded in part by the Spanish Ministerio de Economía y Competitividad and the Agencia Española de Investigación under Grant TEC2016-78434-C3-3-R (AEI/FEDER, EU) and by the Comunidad de Madrid in Spain under Grant S2103/ICE-2845. T. Koch has further received funding from the European Research Council (ERC) under the European Union's Horizon 2020 research and innovation programme (grant agreement number 714161), from the 7th European Union Framework Programme under Grant 333680, and from the Spanish Ministerio de Economía y Competitividad under Grants TEC2013-41718-R and RYC-2014-16332. Pablo M. Olmos has further received funding from the Spanish Ministerio de Economía y Competitividad under Grant IJCI-2014-19150. obtained by spatially-coupling GLDPC block codes with regular DD [6], [7]. With more powerful (compared with simple SPC codes) component codes involved, GLDPC codes have many potential advantages, including improved performance in noisy channels, fast convergence speed and low error floor [4], [8].

So far, GLDPC codes have been designed for a given class of component codes. In this paper, we analyze GLDPC code ensembles using a different approach. Instead of selecting a particular class of component codes and optimizing the graph DD, we are interested in analyzing the tradeoff between coding rate and iterative decoding threshold of ensembles of codes with fixed DD, referred to as the base DD, as we increase the fraction $\nu$ of GC nodes in the graph. Furthermore, our analysis does not consider a particular construction for component codes, but the family of linear block codes of block length $r$ and minimum distance d. The Hamming or sphere-packing bound [9] is used to determine a converse bound on the rate of the GLDPC code ensemble as a function of a triplet of $(\nu, \mathrm{d}, r)$. The Varshamov bound is considered to determine an achievable rate of the GLDPC code ensemble [10].

For the BEC, iterative decoding of graph-based codes, such as LDPC or GLDPC codes, can be performed by means of peeling decoding (PD) algorithms [12], [13], [14]. In the case of GLDPC codes, the asymptotic analysis of PD under ML-decoded component codes (ML-PD, for short) requires the use of multi-edgetype DDs [15] to track down all possible decodable erasure patterns at GC nodes [6], [14]. As a consequence, the list of code parameters to jointly optimize becomes cumbersome. In particular, the parameters include the description of the multiedge $\mathrm{DD}$, the position of GC nodes in the graph, the edge labelling at every GC node used to determine positions in the component block code, and the list of locally ML-decodable erasure patterns. Most works in the literature overcome this problem by either fixing in advance the positions of GC nodes, component codes and decoding method at GC nodes [4], [5], or rely on suboptimal bounded distance (BD) decoding [7], [16], [11].

To simplify the analysis, we propose a probabilistic description of all components of the GLDPC code, namely, the base $\mathrm{DD}$, the presence of GC nodes in the graph, and the decoding method implemented at GC nodes. Regarding the latter aspect, we parameterize the decoding capabilities of a blocklength- $r$ component code by a vector $\mathbf{p}=\left(p_{1}, p_{2}, \ldots, p_{r}\right)$, where $p_{w}$ is the probability that a weight- $w$ erasure pattern chosen at random is decodable, $w \in\{1, \ldots, r\}$. We show how to properly incorporate such a probabilistic description of the decoding capabilities of component codes into the PD algorithm, and 


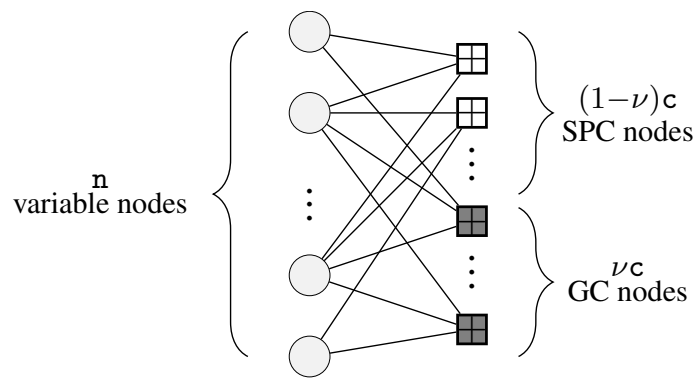

Fig. 1. Tanner graph of a GLDPC code.

denote the resulting algorithm as probabilistic PD (P-PD). Due to its probabilistic nature, the asymptotic analysis of P-PD does not require the use of multi-edge type DDs. By computer simulation, we show that the P-PD performance accurately predicts the actual GLDPC performance when ML decoding is used at GC nodes. We illustrate our analysis for GLDPC code ensembles using $(2,6)$ and $(2,15)$ base DDs. In all cases, we show that a large fraction of GC nodes is required to reduce the original gap to capacity. However, the closest gap to capacity is not achieved at $\nu=1$, but a smaller value must be used. Our results also suggest that the use of very powerful component codes in general does not pay off, since the gain in threshold does not compensate for the severe decrease of the GLDPC code rate. All these results have not been clearly discussed in the literature of GLDPC block codes.

The methodology presented is flexible and decouples the problems of bounding the GLDPC coding rate and the asymptotic analysis of the ensemble. In this regard, broader classes of component codes or improved decoding methods at GC nodes could be incorporated in a systematic way. An extended version of this paper can be found in [17], where we include all derivations and proofs and a larger set of experimental results.

\section{GLDPC ENSEMBLES}

We introduce the GLDPC code ensembles that are analyzed in the rest of the paper and the notation used to define their DD.

\section{A. Degree distribution}

As illustrated in Fig. 1, the Tanner graph of every member in the ensemble contains $\mathrm{n}$ variable nodes (coded bits) and $\mathrm{c}$ parity-check nodes, among which a fraction $\nu$ corresponds to GC nodes while the rest corresponds to SPC nodes. We denote by $\mathrm{E}$ the number of edges in the Tanner graph and we define the degree of a node as the number of edges connected to it.

The DD of the ensemble is characterized as follows. The vector $\bar{\lambda}=\left(\lambda_{1}, \lambda_{2}, \ldots, \lambda_{x}\right)$ is the left $\mathrm{DD}$, where $\lambda_{i}$ represents the fraction of edges (w.r.t. E) connected to a variable of degree $i$. Given $\bar{\lambda}, \mathrm{n}$ and $\mathrm{E}$ are related by [15]

$$
\mathrm{n}=\mathrm{E} \sum_{i=1}^{x} \lambda_{i} / i
$$

The right DD is defined by two vectors $\bar{\rho}_{p}=$ $\left(\rho_{p 1}, \rho_{p 2}, \ldots, \rho_{p r}\right)$ and $\bar{\rho}_{c}=\left(\rho_{c 1}, \rho_{c 2}, \ldots, \rho_{c r}\right)$, where $\rho_{p j}$ denotes the fraction of edges (w.r.t. E) connected to a SPC node that has degree $j$ and $\rho_{c j}$ denotes the fraction of edges (w.r.t.
E) connected to a GC node that has degree $j$. Since the fraction of GC nodes in the graph is $\nu$, the following must hold:

$$
\nu=\frac{\sum_{j=1}^{r} \rho_{c j} / j}{\sum_{u=1}^{r}\left(\rho_{c u}+\rho_{p u}\right) / u} .
$$

For simplicity, we restrict our analysis to the class of GLDPC ensembles characterized by SPC and GC nodes with constant degree $r$. The Tanner graph of any code in this ensemble contains $\mathrm{n}$ variable nodes, $\nu \mathrm{E} / r$ GC nodes and $(1-\nu) \mathrm{E} / r$ SPC nodes. The DD of the GLDPC codes is characterized by the triple $(\bar{\lambda}, r, \nu)$, and the ensemble of codes generated is denoted by $\mathcal{C}_{\bar{\lambda}, r, \nu}$. The DD of the LDPC ensemble obtained by taking $\nu=0$ is defined as the base DD, and the corresponding LDPC code ensemble is referred to as the base ensemble. The coding rate of the base ensemble, denoted by $R_{0}$, is computed as

$$
\mathrm{R}_{0}=1-\frac{1 / r}{\sum_{i=1}^{x} \lambda_{i} / i} .
$$

Finally, we assume that the incoming edges to every degree- $r$ GC nodes are assigned uniformly at random to each position of the component code.

\section{B. The coding rate of the $\mathcal{C}_{\bar{\lambda}, r, \nu}$ ensemble}

Our analysis is not tailored to a specific code construction at component codes, but it considers the family of linear block codes with block length $r$ and minimum distance d. Specifically, we make use of classical results on linear block codes to find bounds on the coding rate of the GLDPC code ensemble.

Lemma 2.1: Let $\mathrm{k}^{(\ell)} \in \mathbb{N}^{+}, \ell=1, \ldots, \nu \mathrm{E} / r$, denote the number of rows in the parity-check matrix associated with the component code of the $\ell$-th GC node. The design rate $\mathrm{R}_{\bar{\lambda}, r, \nu}$ of the $\mathcal{C}_{\bar{\lambda}, r, \nu}$ ensemble is

$$
\mathrm{R}_{\bar{\lambda}, r, \nu}=\mathrm{R}_{0}-\nu\left(1-\mathrm{R}_{0}\right)\left(\mathrm{k}_{\mathrm{avg}}-1\right),
$$

where $\mathrm{k}_{\text {avg }}=\left(\nu \frac{\mathrm{E}}{r}\right)^{-1} \sum_{\ell=1}^{\nu \frac{\mathrm{E}}{r}} \mathrm{k}^{(\ell)}$ denotes the average number of rows in the parity-check matrix of the component codes.

Proof: See [17].

Note that the second term in (4) accounts for the rate loss at GC nodes. The fact that component codes are linear block codes with minimum distance $\mathrm{d}$ can be used to derive the following bounds on the $\mathrm{R}_{\bar{\lambda}, r, \nu}$

Lemma 2.2: If all component codes in the $\mathcal{C}_{\bar{\lambda}, r, \nu}$ ensemble are linear block codes with minimum distance $d>2$, then

$$
\mathrm{R}_{\bar{\lambda}, r, \nu} \leq \mathrm{R}_{0}-\nu\left(1-\mathrm{R}_{0}\right) \log _{2}\left(\frac{1}{2} \sum_{q=0}^{\left\lfloor\frac{\mathrm{d}-1}{2}\right\rfloor}\left(\begin{array}{l}
r \\
q
\end{array}\right)\right) .
$$

Furthermore, there exists a set of linear block codes to be used as component codes such that

$$
\mathrm{R}_{\bar{\lambda}, r, \nu} \geq \mathrm{R}_{0}-\nu\left(1-\mathrm{R}_{0}\right)\left\lceil\log _{2}\left(\frac{1}{2}+\frac{1}{2} \sum_{q=0}^{\mathrm{d}-2}\left(\begin{array}{c}
r-1 \\
q
\end{array}\right)\right)\right] .
$$

Here, we use $\lceil\cdot\rceil$ and $\lfloor\cdot\rfloor$ to denote the ceiling and floor functions, respectively. It is easy to show that the two bounds coincide, for example, when $\mathrm{d}=3$ and $r=2^{z}-1$, where $z \in \mathbb{Z}_{+}$. Thus, in this case the bound in (6) is achieved with equality.

Proof: See [17] 


\section{Probabilistic Peeling Decoding over the BEC}

Suppose we use a random sample of the $\mathcal{C}_{\bar{\lambda}, r, \nu}$ ensemble to transmit over a $\operatorname{BEC}(\epsilon)$. For this channel, each of the $\mathrm{n}$ coded bits is erased with probability $\epsilon$. Without loss of generality, we assume that the all-zero codeword is transmitted, hence the received vector $\boldsymbol{y}$ belongs to the set $\{0, ?\}^{\mathrm{n}}$, where ? denotes an erasure. Let $\Gamma_{\boldsymbol{y}} \subseteq\{1, \ldots, \mathrm{n}\}$ be the index set of the bits correctly received, namely $y_{i}=0$, if $i \in \Gamma_{\boldsymbol{y}}$.

\section{A. Generalized Peeling Decoding}

Decoding will be performed using a generalization of the PD algorithm [12] similar to that proposed for GLDPC codes in [14]. The final formulation of the decoding algorithm depends on the decoding capabilities we assume at GC nodes. While bounded-distance (BD) decoding at component codes considers decodable all GC nodes up to degree $d-1$ [7], [16], in the case of ML decoding we have to specify a full list of decodable erasure patterns and, consequently, label each of the incoming edges at every GC node to differentiate between decodable and non-decodable GC nodes. As shown in [14], incorporating this labelling into the asymptotic analysis requires the use of multiedge type DDs.

\section{B. Probabilistic PD}

In order to incorporate ML decoding at GC nodes into our analysis, and at the same time maintain a formulation compatible with the random definition of the $\mathcal{C}_{\bar{\lambda}, r, \nu}$ ensemble, we assume that the fraction of ML-decodable weight- $w$ erasure patterns at every GC node is given by some $p_{w} \in[0,1], w=$ $1, \ldots, r$. Thus, the family of component codes under analysis is the family of blocklength- $r$ linear block codes with minimum distance $\mathrm{d}$ and with decoding profile described by the vector

\footnotetext{
$\overline{\text { Algorithm } 1 \text { P-PD }}$

Remove from the Tanner graph of the GLDPC code all variable nodes with indexes in $\Gamma_{\boldsymbol{y}}$.

for all GC nodes do

If the GC has degree $w$, tag the check node as decodable with probability $p_{w}$.

\section{end for}

Construct $\Omega$, the index set of check nodes that correspond to either a degree-one SPC node or GC nodes tagged as decodable.

repeat

1) Select at random a member of $\Omega$.

2) Remove from the Tanner graph the check node with the index drawn, all connected variable nodes, and all attached edges.

3)

for every non-decodable GC node that has lost one or more edges do

If the GC has degree $w$, tag the check node as decodable with probability $p_{w}$.

\section{end for}

4) Update $\Omega$.

until All variable nodes have been removed (decoding success) or $\Omega=\emptyset$ (decoding failure).
}

TABLE I

CODING RATES AND ITERATIVE DECODING THRESHOLDS UNDER PD OF DIFFERENT BASE DDS

\begin{tabular}{c|ccccc} 
Base DD & $\bar{\lambda}$ & $r$ & $\mathrm{R}_{0}$ & $\epsilon_{0}$ & Gap to capacity \\
\hline$(2,6)$-regular & $\lambda_{2}=1$ & 6 & $2 / 3$ & 0.206 & 0.127 \\
$(2,15)$-regular & $\lambda_{2}=1$ & 15 & $13 / 15$ & 0.071 & 0.062
\end{tabular}

TABLE II

FAMILIES OF COMPONENT LINEAR BLOCK CODES.

\begin{tabular}{c|cccc} 
Code Family Index & Blocklength $r$ & $\mathrm{~d}$ & $p_{\mathrm{d}}$ & $p_{\mathrm{d}+1}$ \\
\hline I & 6 & 3 & $80 \%$ & 0 \\
II & 6 & 4 & $80 \%$ & 0 \\
III & 15 & 3 & $92.31 \%$ & $61.54 \%$ \\
IV & 15 & 4 & $92.31 \%$ & $61.54 \%$
\end{tabular}

$\mathbf{p}=\left(p_{1}, \ldots, p_{r}\right)$. Note that if the minimum distance of the component code is $\mathrm{d}$, then $p_{w}=1$ for $w \leq \mathrm{d}-1$.

By exploiting the fact that incoming edges at every GC node are randomly assigned to each of the $r$ positions of the component code, we can incorporate ML-decoded GC nodes into PD as shown in Algorithm 1, denoted as probabilistic PD (P-PD). Observe that the key P-PD feature is tagging GC check nodes as decodable with probabilities given by $\mathbf{p}$. Thus, at every iteration, P-PD emulates the ML decoding operation of a degree$w$ GC node by drawing the decoding capability according to a Bernoulli distribution with parameter $p_{w}, w \in\{1, \ldots, r\}$. Note that P-PD must be regarded as a procedure that allows for simpler analysis rather than a practical decoding algorithm. Further, note that we recover the bounded distance PD (BD-PD) algorithm from P-PD if we set $p_{w}=0$ for $w \geq \mathrm{d}$ and $p_{w}=1$ otherwise.

\section{P-PD asymptotic analysis}

In [12] it is shown that if we apply the PD to elements of an LDPC ensemble, then the expected DD of the sequence of residual graphs can be described as the solution to a set of differential equations. The deviation of the process w.r.t. the expected evolution decreases exponentially fast with the LDPC blocklength. This analysis is based on a result on the evolution of Markov processes due to Wormald [18]. In [17] we analyze the asymptotic behaviour of the $\mathcal{C}_{\bar{\lambda}, r, \nu}$ ensemble under P-PD by extending the technique proposed in [12]. An estimate of the PPD threshold for the $\mathcal{C}_{\bar{\lambda}, r, \nu}$ ensemble is obtained by numerically searching for the highest $\epsilon$ value for which there is at least one decodable check node on the residual GLDPC Tanner graph until the end of the decoding process.

\section{ANALYSIS OF THE $\mathcal{C}_{\bar{\lambda}, r, \nu}$ ENSEMBLE UNDER P-PD}

In this section, we study the asymptotic performance of the $\mathcal{C}_{\bar{\lambda}, r, \nu}$ ensemble for different base DDs as we vary the fraction $\nu$ of GC nodes in the graph. We use base DDs that correspond to regular LDPC code ensembles with variable degree equal to 2 , as summarized in Table I. We denote by $\epsilon_{0}$ the PD threshold of the base LDPC ensemble. To find reference block codes as component codes, we exhaustively search over the database [19], [20], which implements MAGMA [21] to design block codes with the largest minimum distance. More precisely, for every $r$, we search for the code with the largest found minimum distance $\mathrm{d}$, and its corresponding $p_{\mathrm{d}}$ and $p_{\mathrm{d}+1}$ parameters. These values are listed in Table II and used as a reference for 


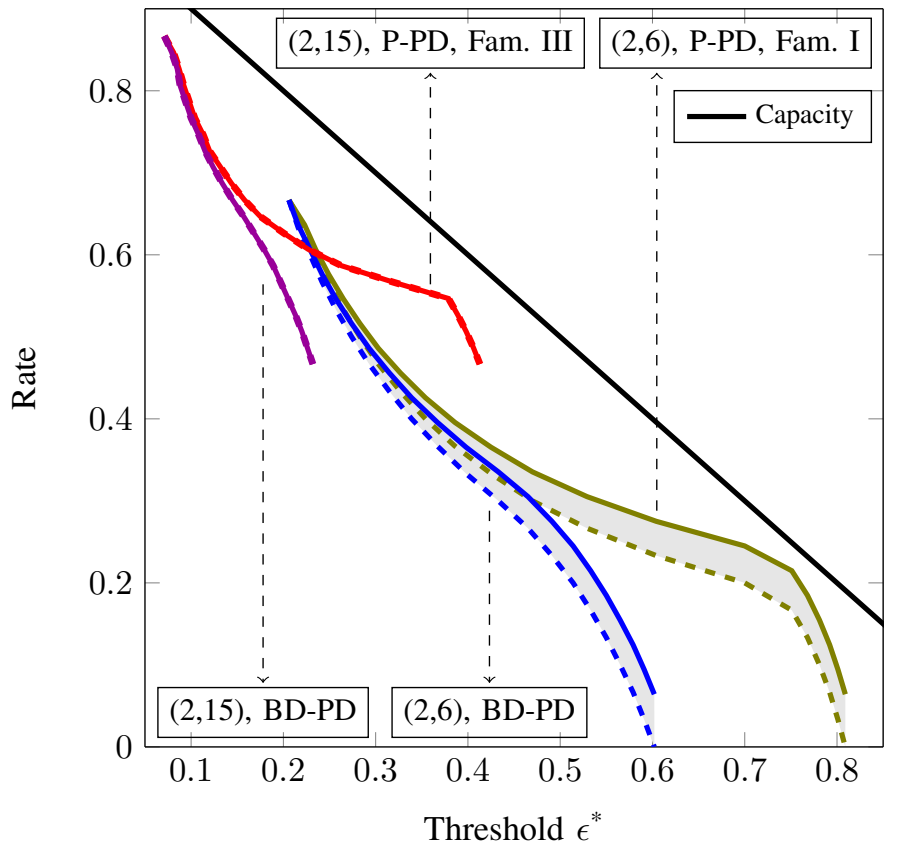

Fig. 2. We compute the bounds on the $\mathcal{C}_{\bar{\lambda}, r, \nu}$ coding rate in (5) and (6) for a base DD $(2,6)$ and $(2,15), \mathrm{d}=3$ component codes as a function of the P-PD and BD-PD thresholds and show the gap to channel capacity. Solid lines represent the converse bound in (5), dashed lines represent the achievable bound in (6), the black line represents the channel capacity.

the whole family of linear block codes. We construct $\mathcal{C}_{\bar{\lambda}, r, \nu}$ ensembles by combining base DDs with the component code families summarized in Table II. For each code ensemble, we compute the P-PD threshold $\epsilon^{*}$ as a function of $\nu$. The inverse of the $\epsilon^{*}(\nu)$ function is denoted by $\nu\left(\epsilon^{*}\right)$ and provides the minimum fraction of GC nodes in the graph required to achieve an ensemble threshold at least $\epsilon^{*}$.

We use Lemma 2.2 to determine bounds on $\mathrm{R}_{\bar{\lambda}, r, \nu}$ for a given targeted decoding threshold $\epsilon^{*}$. By using $\nu\left(\epsilon^{*}\right)$ in (5), we obtain a converse bound on the coding rate required to achieve a $\mathrm{P}$ PD decoding threshold equal to $\epsilon^{*}$ using component codes with minimum distance d. Similarly, using $\nu\left(\epsilon^{*}\right)$ in (6), we obtain an achievable bound on the coding rate required to achieve a PPD decoding threshold equal to $\epsilon^{*}$ using linear component codes with minimum distance $d$. We proceed along the same lines to obtain bounds on the $\mathcal{C}_{\bar{\lambda}, r, \nu}$ rate for the BD-PD thresholds.

In Fig. 2 we plot these bounds for both P-PD and BD-PD using families of block component codes I and III, both with minimum distance $d=3$. Observe first that the performance oft both BD-PD and P-PD approximately overlaps for coding rates close to the original rate of the base $\mathrm{DD}$, i.e., for small values of $\nu .{ }^{1}$ However, while the BD-PD gap to capacity always grows with $\nu$, this is not the case for P-PD. Furthermore, there are values of $\nu$ for which the P-PD gap to capacity is smaller than for than the base LDPC ensemble under PD. For the $(2,6)$ base DD, the P-PD minimum gap to capacity, measured using the achievable rate bound, is 0.0823 for a coding rate of 0.2254 . According to Table I, for this base DD, the PD gap to capacity is 0.127 . For the $(2,15)$ base DD with Fam. III block component codes, observe that the two rate bounds coincide, as predicted by Lemma 2.2. In this case, the P-PD mimimum gap to capacity

\footnotetext{
${ }^{1}$ Recall that by (4), increasing $\nu$ yields smaller coding rates.
}

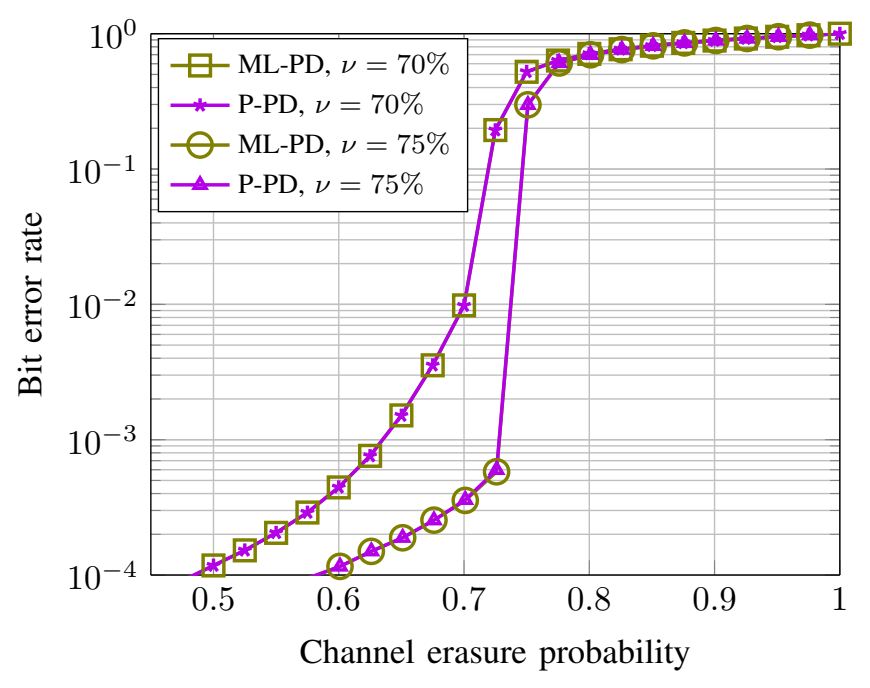

Fig. 3. Simulation results for the $\mathcal{C}_{\bar{\lambda}, r, \nu}$ ensemble with $(2,6)$ base DD, code length 10002 and Code I. We compare P-PD decoding using the corresponding parameters in Table II with ML-decoded GC nodes using Hamming $(6,3)$ code with $\mathrm{d}=3$

is 0.0743 , achieved at a coding rate of 0.5476 .

\section{Selecting SPECIFIC COMPONENT CODES}

By using the bounds on the $\mathcal{C}_{\bar{\lambda}, r, \nu}$ code rate, we have been able to assess the performance of the $\mathcal{C}_{\bar{\lambda}, r, \nu}$ ensemble for a whole family of linear block component codes. In certain scenarios the proposed bounds on the $\mathcal{C}_{\bar{\lambda}, r, \nu}$ code rate provide meaningful design information about the asymptotic behaviour of the ensemble. The natural question that arises at this point is whether we can find specific component codes within the family that improve the achievability bound in (6), reducing the gap to the rate converse bound in (5). In this section, we analyze the asymptotic performance of the $\mathcal{C}_{\bar{\lambda}, r, \nu}$ ensemble when we use the specific block component codes in Table III. The construction of these linear block codes is detailed in [19], and their the generator matrix is given in the extended version of this manuscript [17]. Upon selecting a specific component code, we can compare by simulation the $\mathcal{C}_{\bar{\lambda}, r, \nu}$ performance under P-PD with the performance obtained when PD is combined with GC nodes decoded via ML. As an example, in Fig. 3 we present the simulated average performance of members of the $\mathcal{C}_{\bar{\lambda}, r, \nu}$ ensemble for a $(2,6)$ base DD with Code A component codes. The perfect match between ML-PD decoder and P-PD in all cases shows that we are not sacrificing accuracy with the probabilistic description of the decoder.

Once we fix a particular class of component codes to be used at GC nodes, we can replace the $\mathcal{C}_{\bar{\lambda}, r, \nu}$ code bounds by the actual code rate in (4). In Fig. 4 we plot the $\mathcal{C}_{\bar{\lambda}, r, \nu}$ coding rate and the achievable bound of the corresponding family of codes for $(2,6)$ and $(2,15)$ base DDs as a function of the P-PD threshold. Observe that with the component codes proposed, we are able to perform at least as good as the achievable bound of the corresponding family of block component codes. Note also that in the case of the $(2,6)$ base $\mathrm{DD}$, the use of component codes of type $\mathrm{B}$, which have higher mimimum distance than codes of type A but also lower coding rate, does not improve the GLDPC asymptotic performance. 
TABLE III

BLOCK COMPONENT CODES. $k$ IS THE NUMBER OF ROWS IN THE CODE PARITY-CHECK MATRIX.

\begin{tabular}{c|cccc} 
Code Index & Blocklength $r$ & $\mathrm{k}$ & Rate & Code Family \\
\hline A & 6 & 3 & $1 / 2$ & I \\
B & 6 & 4 & $1 / 3$ & II \\
C & 15 & 4 & $11 / 15$ & III \\
D & 15 & 5 & $2 / 3$ & IV
\end{tabular}

\section{Conclusions And Future Lines of Research}

One of the main contributions of the presented paper is the methodology itself. By introducing a certain amount of GC nodes in the LDPC Tanner graph, we analyze if the gap to channel capacity is reduced at the resulting coding rate. We have also proposed the P-PD algorithm as a flexible model to analyze beyond-BD decoding algorithm at GC nodes. Note that in our analysis, the evaluation of both coding rate and of iterative decoding threshold are decoupled problems. In this regard, broader classes of component codes or improved decoding methods at GC nodes could be incorporated in a systematic way. In addition, one of our main lines of research is the extension of the proposed analysis of GLDPC ensembles to incorporate rate adaptation techniques by means of puncturing [22] and the use of generalized variable nodes [23].

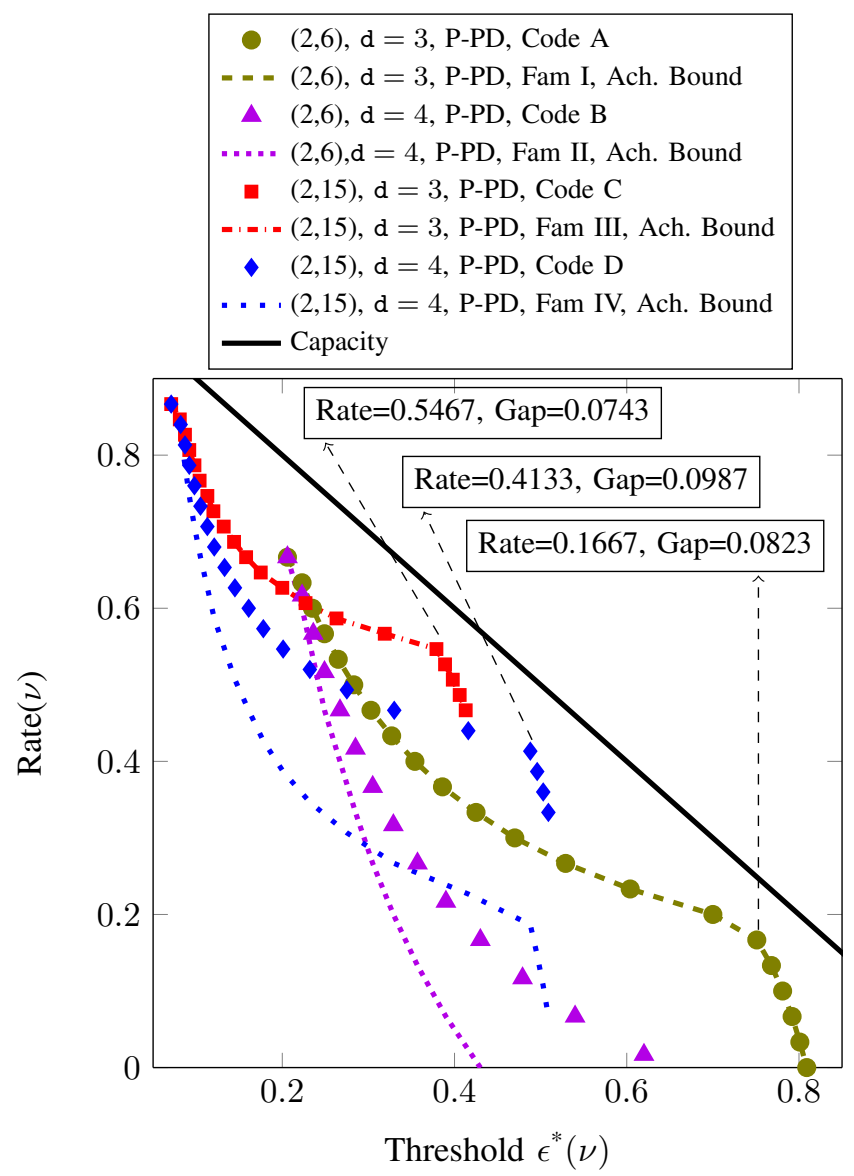

Fig. 4. We compute the $\mathcal{C}_{\bar{\lambda}, r, \nu}$ coding rate and the achievable bound of the corresponding family of codes for $(2,6)$ and $(2,15)$ base DDs as a function of the P-PD threshold.

\section{REFERENCES}

[1] R. Tanner, "A recursive approach to low complexity codes," IEEE Trans. on Inf. Theory, vol. 27, no. 5, pp. 533 - 547, Sept. 1981.

[2] M. Lentmaier and K. Zigangirov, "On generalized low-density paritycheck codes based on Hamming component codes," IEEE Communications Letters, vol. 3, no. 8, pp. 248-250, Aug 1999.

[3] G. Yue, L. Ping, and X. Wang, "Generalized Low-Density Parity-Check Codes Based on Hadamard Constraints," IEEE Trans. on Inf. Theory, vol. 53, no. 3, pp. 1058-1079, March 2007.

[4] G. Liva, W. Ryan, and M. Chiani, "Quasi-cyclic generalized LDPC codes with low error floors," IEEE Trans. on Communications, vol. 56, no. 1, pp. 49-57, January 2008.

[5] S. Abu-Surra, D. Divsalar, and W. E. Ryan, "Enumerators for protographbased ensembles of LDPC and generalized LDPC codes," IEEE Trans. on Inf. Theory, vol. 57, no. 2, pp. 858-886, Feb. 2011.

[6] M. Lentmaier and G. Fettweis, "On the thresholds of generalized LDPC convolutional codes based on protographs," in Proc. IEEE ISIT, Austin, USA., June 2010, pp. 709-713.

[7] Y. Y. Jian, H. D. Pfister, and K. R. Narayanan, "Approaching capacity at high rates with iterative hard-decision decoding," in Proc. IEEE ISIT, Boston, USA, July 2012, pp. 2696-2700.

[8] D. Mitchell, M. Lentmaier, and D. Costello, "On the minimum distance of generalized spatially coupled LDPC codes," in Proc. IEEE ISIT, Istanbul, Turkey, July 2013, pp. 1874-1878.

[9] F. J. MacWilliams and N. J. A. Sloane, The theory of error correcting codes. North-Holland Pub. Co. New York, 1977.

[10] W. C. Huffman and V. Pless, Fundamentals of error-correcting codes. Cambridge, U.K., New York: Cambridge University Press, 2003.

[11] Paolini, E. and Fossorier, M.P.C. and Chiani, M., "Generalized and Doubly Generalized LDPC Codes With Random Component Codes for the Binary Erasure Channel," IEEE Trans. on Inf. Theory, vol. 56, no. 4, pp. 16511672, April 2010.

[12] M. Luby, M. Mitzenmacher, M. Shokrollahi, and D. Spielman, "Efficient erasure correcting codes," IEEE Trans. on Inf. Theory, vol. 47, no. 2, pp. $569-584$, Feb. 2001.

[13] C. Measson, A. Montanari, and R. Urbanke, "Maxwell construction: The hidden bridge between iterative and maximum a posteriori decoding," IEEE Trans. on Inf. Theory, vol. 54, no. 12, pp. 5277 -5307, Dec. 2008.

[14] P. Olmos, D. Mitchell, and J. Costello, D.J., "Analyzing the finite-length performance of generalized LDPC codes," in Proc. IEEE ISIT, Hong Kong, China, June 2015, pp. 2683-2687.

[15] T. J. Richardson and R. Urbanke, Modern Coding Theory. Cambridge University Press, Mar. 2008.

[16] N. Miladinovic and M. Fossorier, "Generalized LDPC codes and generalized stopping sets," IEEE Trans. on Communications, vol. 56, no. 2, pp. 201-212, February 2008.

[17] Y. Liu, P. Olmos, and T. Koch, "On LDPC Code Ensembles with Generalized Constraints," Online available at http://www.tsc.uc3m.es/ olmos/Drafts.html, 2017.

[18] N. C. Wormald, "Differential equations for random processes and random graphs," Annals of Applied Probability, vol. 5, no. 4, pp. 1217-1235, 1995.

[19] M. Grassl, "Bounds on the minimum distance of linear codes and quantum codes," Online available at http://www.codetables.de, 2007, accessed on 2017-01-07.

[20] _ _ "Searching for linear codes with large minimum distance," in Discovering Mathematics with Magma - Reducing the Abstract to the Concrete, ser. Algorithms and Computation in Mathematics, W. Bosma and J. Cannon, Eds. Heidelberg: Springer, 2006, vol. 19, pp. 287-313.

[21] W. Bosma, J. Cannon, and C. Playoust, "The Magma Algebra System I: The User Language," Journal of Symbolic Computation, vol. 24, no. 3-4, pp. 235-265, Oct. 1997.

[22] D. Mitchell, M. Lentmaier, A. Pusane, and D. Costello, "Randomly Punctured LDPC Codes," IEEE Journal on Selected Areas in Communications, vol. 34, no. 2, pp. 408-421, Feb 2016

[23] Y. Wang and M. Fossorier, "Doubly Generalized LDPC Codes over the AWGN Channel," IEEE Trans. on Communications, vol. 57, no. 5, pp. 1312-1319, May 2009. 